\title{
Equilibrium, Kinetics and Thermodynamic Studies on the Removal of Safranin-O from Waste Water Using Activated Carbons Derived from Coconut Shell and Pineapple Peel
}

\author{
Jibrin Mohammed ${ }^{1, ~ *, ~ U f a r u n a ~ I d r i s u ~ N o a h ~}{ }^{1}$, Isah Jibrin ${ }^{1}$, Kabiru Suleiman Madaki \\ Stephen Ingedu Audu ${ }^{1}$ \\ ${ }^{1}$ Department of Chemistry, Faculty of Natural \& Applied Sciences, Nasarawa State University, Keffi, Nigeria \\ ${ }^{2}$ Department of Science Laboratory Technology, Faculty of Natural \& Applied Sciences, Nasarawa State University, Keffi, Nigeria
}

Email address:

jibrilmohammed50@gmail.com (J. Mohammed)

${ }^{*}$ Corresponding author

\section{To cite this article:}

Jibrin Mohammed, Ufaruna Idrisu Noah, Isah Jibrin, Kabiru Suleiman Madaki, Stephen Ingedu Audu. Equilibrium, Kinetics and Thermodynamic Studies on the Removal of Safranin-O from Waste Water Using Activated Carbons Derived from Coconut Shell and Pineapple Peel. American Journal of Physical Chemistry. Vol. 8, No. 1, 2019, pp. 1-10. doi: 10.11648/j.ajpc.20190801.11

Received: December 14, 2018; Accepted: January 9, 2019; Published: January 30, 2019

\begin{abstract}
This work is aimed at comparing the adsorption capacities of the activated carbons derived from coconut shell and pineapple peels using Safranin-O as adsorbate. Physical activation method was employed to generate the activated carbons using phosphoric acid $\left(\mathrm{H}_{3} \mathrm{PO}_{4}\right)$ as activating agent. Batch adsorption experiment was employed for the adsorption process. Effects of experimental factors such as adsorbent dosage, inititial Safranin-O dye concentration, $\mathrm{pH}$ and contact time on the adsorption process were examined. The results showed that the adsorption capacities were dependent on these factors. Langmuir and Freundlich Isotherms Models were applied and the results indicated that both models fitted well with the observed data but Freundlich Model fitted better. Pseudo first and second order kinetic models were also applied to describe the adsorption kinetic, only the second order model fitted well with the experimental data. Thermodynamic parameters such as enthalpy change $(\Delta H)$, Gibb's free energy change $(\Delta G)$ and entropy change $(\Delta S)$ were evaluated using Van't Hoff equations, both the free energy and enthalpy change were found to be negative indicating the feasibility and exorthermic nature of the adsorption process respectively. While the entropy change was found to be positive indicating that the degree of dispersion in the adsorption process increased with increase in temperature, Based on the results obtained from this research work, the activated carbon derived from coconut shell (CSAC) showed better adsorbent when compared with the one derived from pineapple peel (PPAC) as CSAC recorded the highest percentage of Safranin-O dye removal from waste water under all the experimental conditions.
\end{abstract}

Keywords: Safranin-O, Removal, Activated Carbon, Coconut Shell, Pineapple Peel

\section{Introduction}

Today, the applications of dyes in various chemical industries have no doubt caused a significant negative impact in the environment, especially on water bodies. However, dyes are considered so important in chemical industries such as textile, printing pharmaceutical, ink, rubber, plastics, leather, cosmetics, and food industries that without them production cannot be complete[1]. This is because they are mostly used in coloring their products which make them attractive. Consequently, they become common source of industrial pollutants during their synthesis and later during their applications in these industries, especially the textile industries [2]. For instance, studies have shown that textile industry is the largest consumer of dye stuffs, it is estimated that $20 \%$ of dyes produced annually are discharged as effluents from manufacturing operations whilst approximately $40-65 \%$ are discharged from textile and associated industries [3].

However, indiscriminate discharge of waste water into water bodies without proper treatment from these industries as byproducts has been reported to have negative impact on 
the normal function of aquatic lives and change in food web [4].

Dyes are broadly classified as anionic, cationic and nonionic depending on the ionic charge on the dye molecules. Investigations have shown that cationic dyes are more toxic than anionic dyes [5]. In the present study, Safranin-O was used as a case study. Safranin-O is a cationic dye mostly use in textile and pharmaceutical industries. Literature reveals that exposure to these effluents may be irritating to respiratory systems, skin, and digestive tract infections when ingested [6].

It is therefore, imperative to decolorize waste water to the lowest permissible concentration in order to safeguard the water bodies as stated by environmental regulations.

The different methods of conventional waste water treatment includes oxidation or ozonation, membrane separation, precipitation, coagulation/flocculation, ionexchange, and reverse osmosis [6]. However, a lot of findings have indicated that it is challenging using conventional methods to effectively remove dye from waste water because of their synthetic origin and complex nature of structures mainly aromatic structures, which are biologically non-degradable and may be toxic to health $[6,7,8]$.

In order to remove dye from industrial effluents, several processes have been adopted by researchers worldwide. Among several chemical and physical methods available, the adsorption process has been reported as one of the most effective techniques that has been successfully employed for colour removal from waste water [9]. However, commercial activated carbon is one of the most widely used agents of decontaminating or decolorizing waste water due to its high adsorption capacity, surface area and degree of surface reactivity as well as micro porous structures [10]. Also, its effectiveness in yielding good results after treatment has been proven [11].

However, economically, the cost of using commercial activated carbon is very high as such researchers developed an alternative low-cost adsorbents that can compete favorably with commercial activated carbons among which are agricultural wastes which have been proven to be effective, green and more economical method that can alternate the more expensive commercially available activated carbons $[11,12]$.

Furthermore, studies have shown that consumption of fruits such as cocoa nut, banana, orange and pineapple fruits is increasng on daily basis which was attributed to the fact that they have high nutritional and medicinal values. Consequently, they also generate a significant amount of waste into the environment [13]. Therefore, their conversion into adsorbents such as activated carbon is a wise decision.

\section{Materials and Methods}

\subsection{Preparation of Adsorbate}

Safranin-O (cationic red dye; chemical formula, $\mathrm{C}_{20} \mathrm{H}_{19} \mathrm{CIN}_{4}$; $\mathrm{MW}, 350.84 \mathrm{~g} \mathrm{~mol}^{-1}$; IUPAC name as 3, 7- diamino-2, 8-dimethyl-5-phenylphenazinium chloride) of high analytical grade was used as an adsorbate. The chemical was purchased in Jos, Plateau State, Nigeria. A stock solution of $1000 \mathrm{ml}$ was prepared by dissolving $1.0 \mathrm{~g}$ of Safranin-O in $1000 \mathrm{ml}$ of distilled water. Different concentrations were prepared by diluting the stock solution with suitable volume of distilled water and the natural $\mathrm{pH}$ of the stock solution was determined using $\mathrm{pH}$ meter (Jenway 3015).<smiles>Cc1cc2nc3cc(C)c(N)cc3[n+](-c3ccccc3)c2cc1N</smiles>

Figure 1. Molecular Structure of Safranin-O (BR2).

\subsection{Preparation of Activated Carbon}

Samples of the coconut shells and pineapple peels were collected from fruits sellers in Keffi local government area of Nasarawa State, Nigeria. The dried samples were ground and sieved to $2 \mathrm{~mm}$ mesh size. Physical activation method (two step process) which involves carbonization and activation was employed.

\subsubsection{Carbonization}

The pretreated $100 \mathrm{~g}$ of each sample $(2 \mathrm{~mm}$ mesh size) was placed in a separate crucible and introduced into a muffle furnace at $500^{\circ} \mathrm{C}$ for ten (10) minutes after which the content was transferred from the crucible into a bath of ice block. The excess water was drained and the samples were sun dried. The carbonized sample was washed with $10 \% \mathrm{HCl}$ to remove surface ash, followed by hot water washing and rinsing with distilled water to remove residual acid [13]. The solid particles were then sun dried and finally dried in the oven at $100^{\circ} \mathrm{C}$ for $1 \mathrm{hr}$.

\subsubsection{Activation}

Five grams $(5 \mathrm{~g})$ of each carbonized sample was accurately weighed and separately mixed with $5 \mathrm{ml}$ of $1 \mathrm{M}$ activating agent $\left(\mathrm{H}_{3} \mathrm{PO} 4\right)$, the samples were kept for $24 \mathrm{hrs}$ after which they were introduced into a muffle furnace at $800^{\circ} \mathrm{C}$ and maintained for $5 \mathrm{mins}$. The activated carbon produced from each sample was cooled in a cold water, drained off and further washed with $10 \% \mathrm{HCl}$ to remove surface ash, followed by hot water washing and rinsing with distilled water to remove residual acid, sun dried, and finally dried in an oven at $105^{\circ} \mathrm{C}$ for two hours [14]. The prepared activated carbons were then stored in air tight containers for further studies.

\subsection{Adsorption Experiments}

Batch adsorption experiments were employed for the removal of Safranin-O dye from the solution. A predetermined amount $(0.2 \mathrm{~g})$ of each adsorbent was added 
into $100 \mathrm{ml}$ conical flasks each containing $30 \mathrm{ml}$ of Safranin-O dye solution of varying concentrations $(10-50 \mathrm{mg} / \mathrm{L})$ while other factors were kept constant. The contents were placed in an orbital shaker and thoroughly shaken at constant speed of $250 \mathrm{rpm}$ at ambient temperature. One milliliter $(1 \mathrm{ml})$ of each sample was withdrawn from the batch adsorption system at predetermined time intervals (30minutes) with the use of a micro-pipette (Dragon-Med Pipet-tor) and the supernatant was centrifuged at a speed of $400 \mathrm{rpm}$ for $10 \mathrm{~min}$. in order to separate the used adsorbent from the remaining dye solution Similarly, in order to determine the optimal conditions for the Safranin-O adsorption, other factors such as the amount of adsorbent (0.2-1.0 g), $\mathrm{pH}$ (2-10), adsorption temperature, $\left(30-60^{\circ} \mathrm{C}\right)$ and adsorption time $(20-100 \mathrm{~min}$.) were studied using the same method described above.

The unabsorbed dye absorbance characteristics were determined using UV/visible spectrophotometer (6100) at the maximum absorbance wavelength of $520 \mathrm{~nm}$. The absorbance values found were used to estimate the corresponding concentrations using the calibration curve[14].

All the experiments were carried out in duplicates and the average values of the results were used to compute the amount of Safranin-O adsorbed at equilibrium $\mathrm{Q}_{\mathrm{e}}$, the amount adsorbed at any time $\mathrm{t}, \mathrm{Q}_{\mathrm{t}}(\mathrm{mg} / \mathrm{g})$ and the percentage removal $(\% \mathrm{RE})$ using equations $(1,2 \& 3)$ respectively [15].

$$
\begin{gathered}
Q_{e}=\frac{\left(c_{o}-c_{t}\right) V}{W} \\
Q_{t}=\frac{\left(c_{o}-c_{t}\right) V}{W} \\
\% \mathrm{RE}=\frac{\left(c_{o}-c_{e}\right)}{C_{o}} \times 100
\end{gathered}
$$

\section{Results and Discussion}

\subsection{Effect of Initial Concentration}

The effect of varying concentration $(10-50 \mathrm{mg} / \mathrm{L})$ of Safranin-O dye in equoes solution using a fixed mass of the adsorbents $(0.2 \mathrm{~g})$ from coconut shell activated carbon (CSAC) and pineapple peels activated carbon (PPAC) was studied. It could be observed from both figures 2 and 3 shown below that as the concentration of the Safranin-O increases from $10 \mathrm{mg} / \mathrm{L}$ to $50 \mathrm{mg} / \mathrm{L}$, the percentage removal decreases from $96.40-60.28 \%$ for CSAC and $89.50-67.25 \%$ for PPAC respectively. This implies that the higher the concentration of the Safranin-O dye in the solution, the lower the percentage of dye removed or adsorbed. This may be due to the saturation of adsorption sites on the adsorbent surface as the concentration increases. At low concentrations, there will be unoccupied active sites on the adsorbent surface, and when the initial dye concentration increases, the active sites require for adsorption of the dye molecules will not be available. It could also be seen that the percentage removal in coconut shell adsorbent is a little bit higher than in pineapple peels, this could be attributed to the fact that the active sites and the porosity of the coconut shell adsorbent are more than that of the pineapple peels [16].

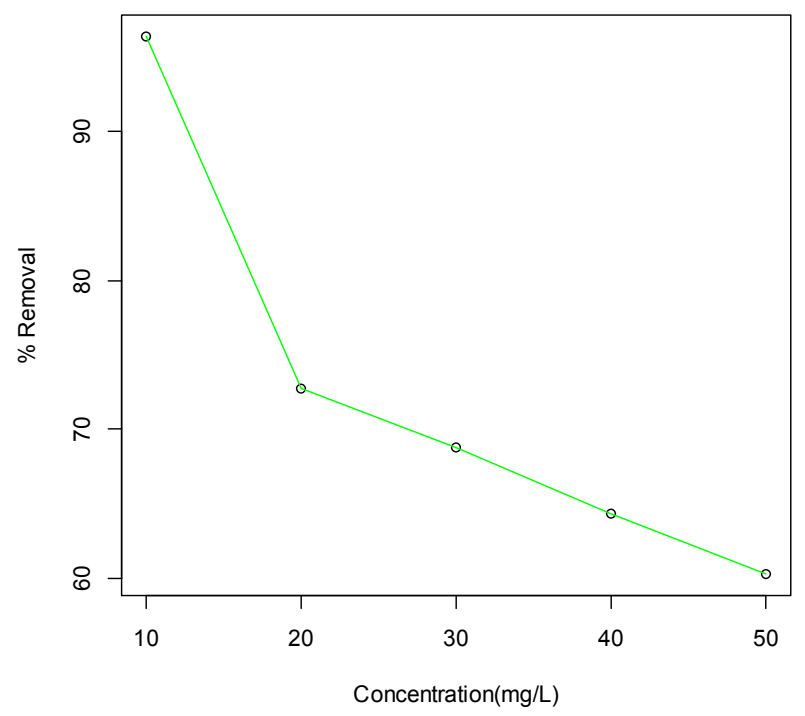

Figure 2. Effect of Initial Concentration Using CSAC.

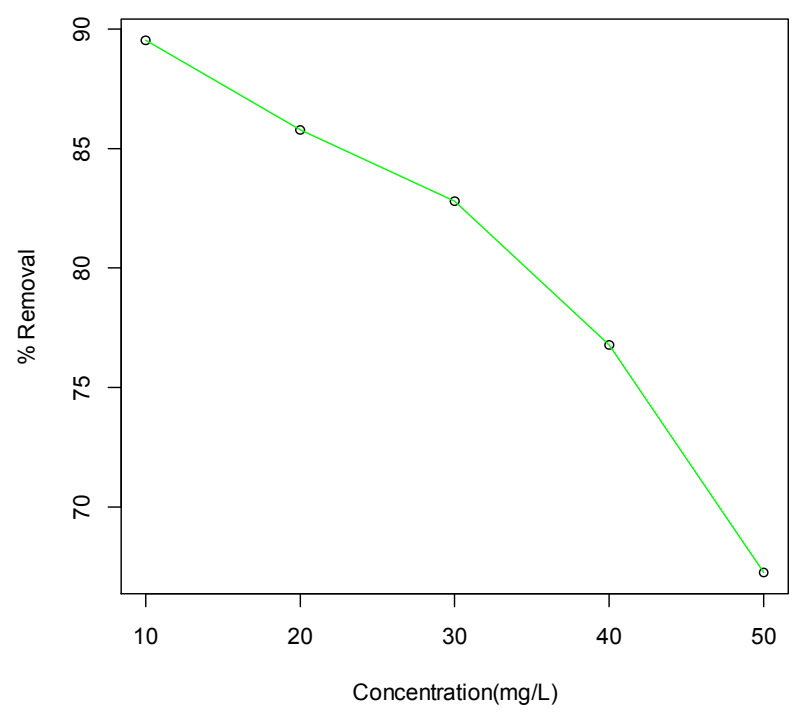

Figure 3. Effect of Initial Concentration of Safranin-O Dye Using PPAC.

\subsection{Effect of Adsorbent Dosage (g)}

Adsorbent dosage plays a vital role in adsorption studies because it also determines the adsorption capacity of the adsorbent for a given initial concentration of dye solution [17]. The results from the figures 4 and 5 reveal that as the adsorbent dosage increases, the percentage removal of Safranin-O dye from the solution also increases from 0.20 $0.60 \mathrm{~g}$ for CSAC and $0.20-0.80$ for PPAC after which no significant changes in the percentage of Safranin-O dye removal were observed with futher increase in the adsorbents dosage. This implies that CSAC and PPAC attained adsorption equilibrium at the dosage of $0.60 \mathrm{~g}$ while PPAC attained adsorption equilibrium with $(0.80 \mathrm{~g})$ dosage. Literature reveals that the efficiency of dye removal increases with increase in adsorption sites of the adsorbent and 
decreases with decrease in the adsorption sites of the adsorbents[18, 19, 20].

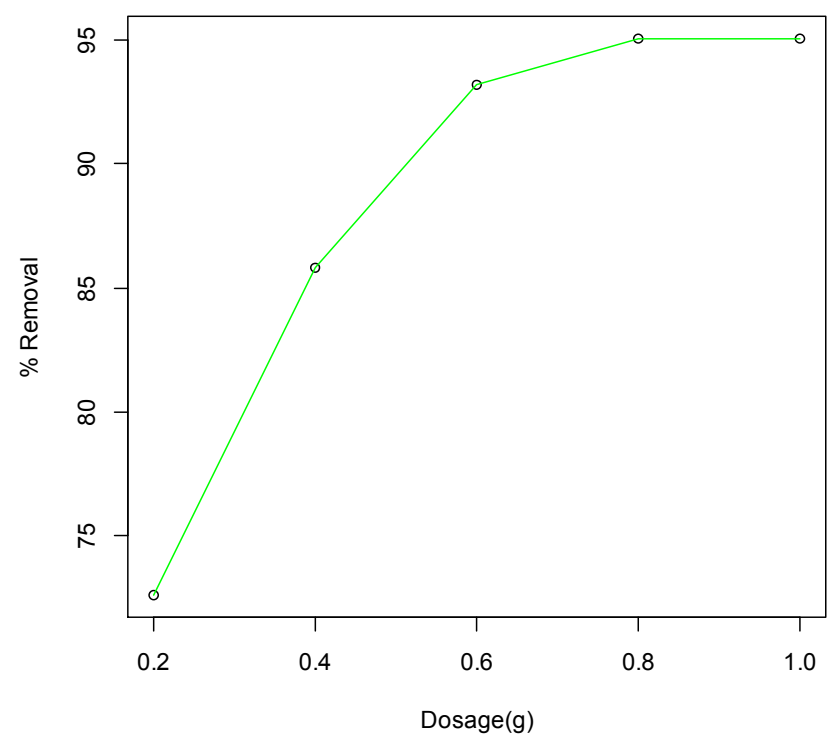

Figure 4. Effect of Dosage on Safranin-O Using CSAC.

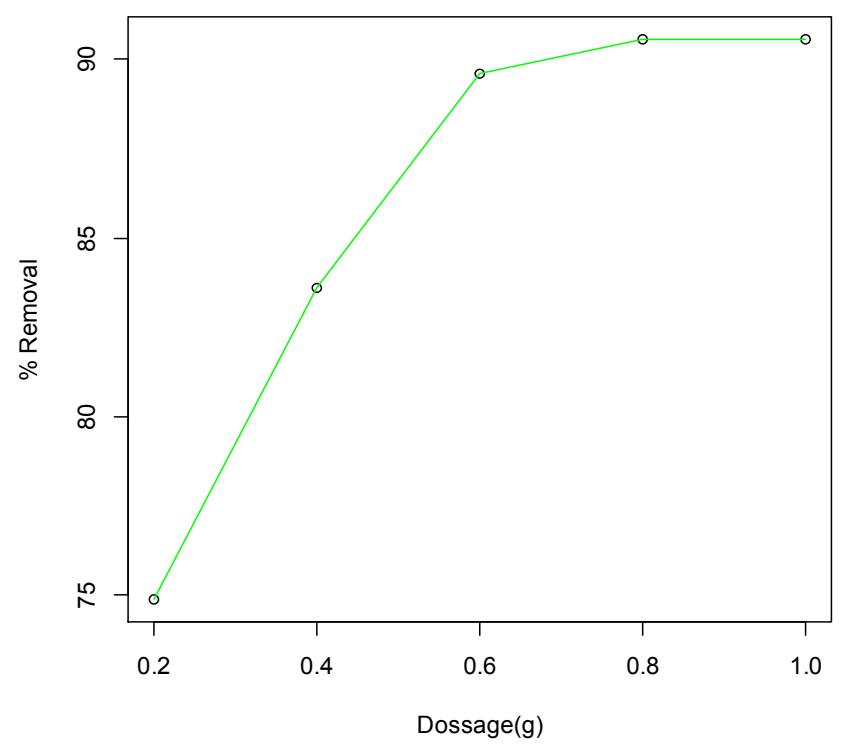

Figure 5. Effect of Dosage on Adsorption of Safranin-O Using PPAC.

\subsection{Effect of Contact Time}

The contact time was found to play an important role in the adsorption process of Safranin-O onto the two derived activated carbons; CSAC and PPAC as shown in figures 6 and 7 respectively. The results of the investigation reveals that percentages of the Safranin-O removed were between the ranges of $59.95-90.70 \%$ for CSAC and $50.65-78.88 \%$ for PPAC respectively. The rapid removal of Safranin-O dye and establishment of equilibrium within a short period indicate the efficiency of the adsorbents for their use in wastewater treatment [21]. It could be seen that removal of Safranin-O dye reached maximum at 80 mins for both CSAC and PPAC. This implies that both CSAC and PPAC attained maximum adsorption equilibrium at the same time. But, the CSAC recorded higher percentage removal which could be attributed to the differences in the number of their active sites and porosity.

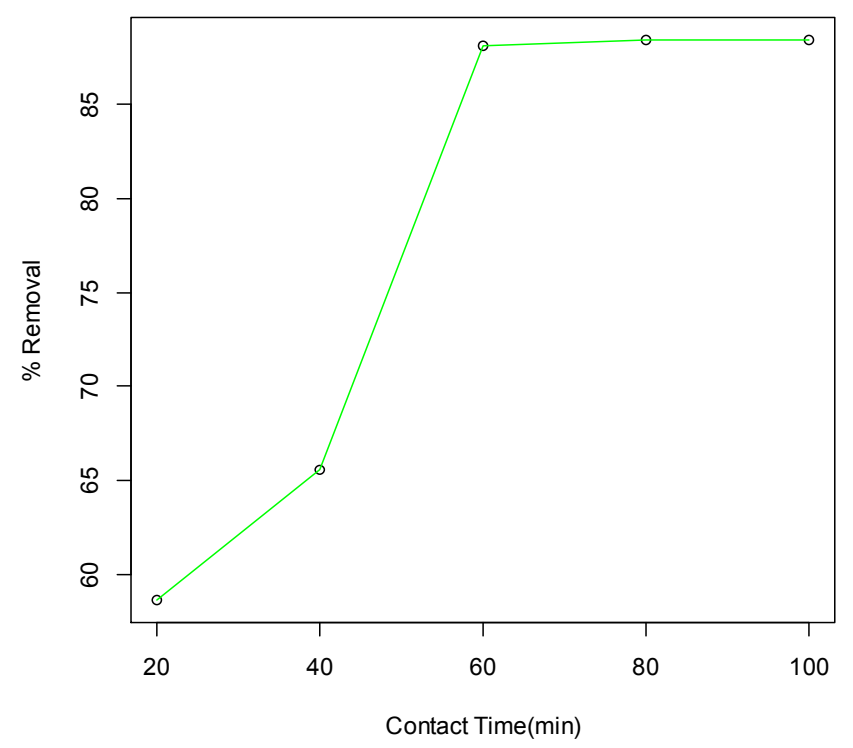

Figure 6. Effect of Contact Time on Adsorption of Safranin-O Using PPAC.

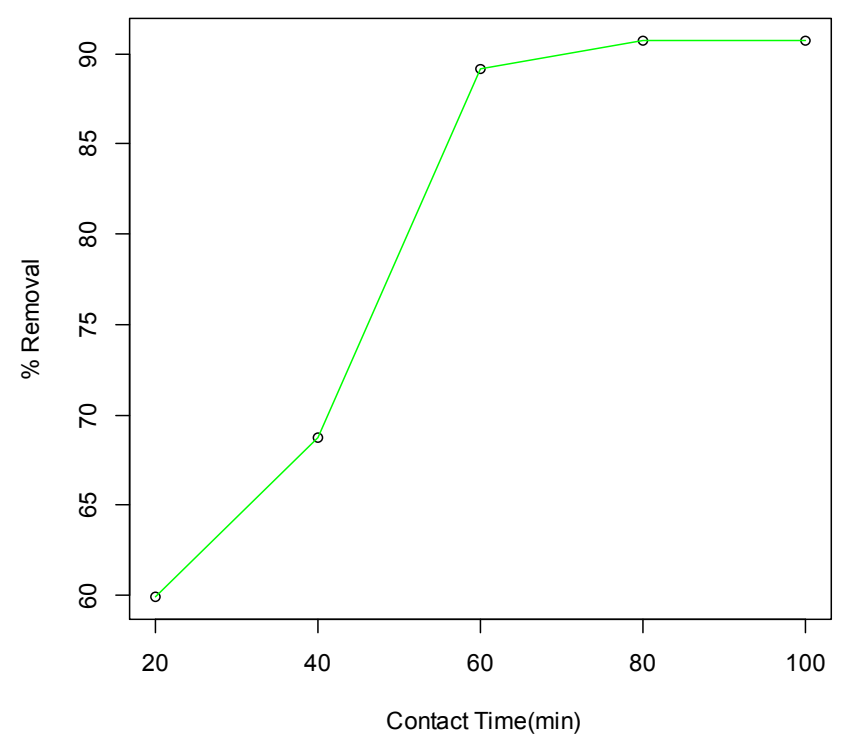

Figure 7. Effect of Contact Time on Adsorption of Safranin-O Using CSAC.

\subsection{Effect of $\mathrm{pH}$}

$\mathrm{pH}$ is a very crucial factor in adsorption studies especially on the adsorption of dyes because it controls the magnitude of electrostatic charges which are imparted both by the ionized dye molecules and the charges on the surface of the adsorbent. As a result, rate of adsorption varies with both the $\mathrm{pH}$ of an adsorbate and the adsorbent [22]. Figures 8 and 9 show the effect of varying $\mathrm{pH}(2,4,6,8$ and 10$)$ on the adsorption efficiency of the derived adsorbents. The results reveal that the lowest and highest percentage of Safranin-O dye removed were observed at the $\mathrm{pH}$ of 2 and 10 for both CSAC and PPAC respectively. This implies that increase in $\mathrm{pH}$ of the Safranin-O dye solution also increased the 
percentage of Safranin-O dye removed. This could be as a result of the fact that as the $\mathrm{pH}$ of a system decreases, the number of negatively charged adsorbent sites also decreases while the number of positively charged surface sites increases. Also, as the $\mathrm{pH}$ of a system increases, the number of negatively charged surface sites increases and the number of positively charged surface sites decreases [22].

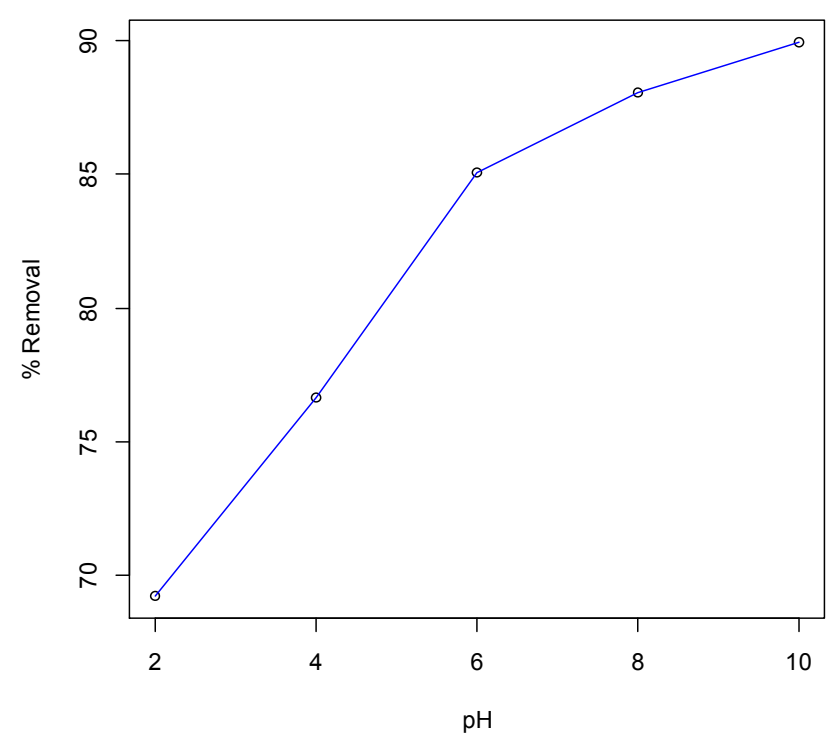

Figure 8. Effect of pH on Adsorption of Safranin-O Using PPAC.

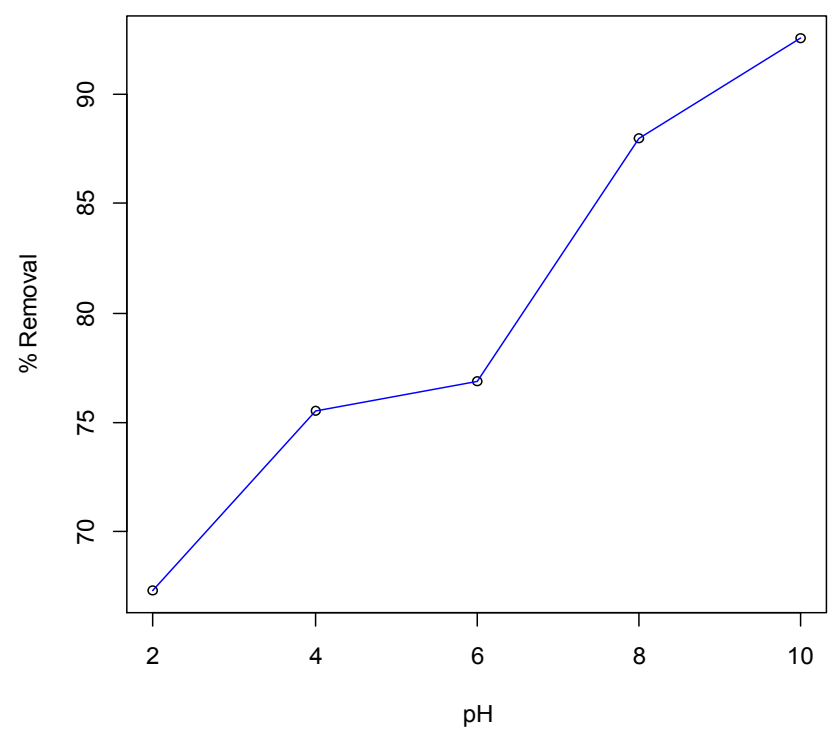

Figure 9. Effect of pH on Adsorption of Safranin-O Using CSAC.

\subsection{Adsorption Isotherms Analysis}

Adsorption isotherm models provide important information use to identify and describe the nature of the adsorption behavior between the adsorbate and adsorbent in the adsorption process [23]. In this study, Langmuir and Freundlich adsorption isotherm models were applied for the adsorption isotherm study. The linearised form of Langmuir isotherm equation (4) was applied to characterize the adsorption process of Safranin-O onto the adsorbents[24 ].

$$
\frac{1}{Q_{e}}=\frac{1}{Q_{\max }}+\left(\frac{1}{B Q_{\max }}\right)\left(\frac{1}{c_{e}}\right)
$$

Where $Q_{e}$, is the equilibrium dye concentration on adsorbent at any time t $(\mathrm{mg} / \mathrm{g}), Q_{\max }$ is the maximum Langmuir monolayer adsorption capacity $(\mathrm{mg} / \mathrm{g}), B$ is Langmuir constant $(\mathrm{L} / \mathrm{mg})$ and $\mathcal{C}_{e}$ is the equilibrium dye concentration in the solution $(\mathrm{mg} / \mathrm{L})$. The Langmuir constant $\mathrm{B}$, was used to determine the suitability or favourability of the adsorption process using the Hall separation factor $\left(\mathrm{R}_{\mathrm{L}}\right)$ as follows:

$$
\mathrm{R}_{\mathrm{L}}=\frac{1}{1+B C_{0}}
$$

Where $\mathrm{R}_{\mathrm{L}}$ is the constant separation factor (dimensionless) and can be used for interpretation of the sorption type as described; $\mathrm{RL}>1$, unfavorable $\mathrm{RL}<0$, unfavorable $\mathrm{RL}=1$, favorable (linear) $0<\mathrm{RL}<1$, favorable and $\mathrm{RL}=0$, irreversible.

The linearised form of Freundlich isotherm is shown in equation (6).

$$
\log \mathrm{Q}_{\mathrm{e}}=\log k_{f}+\left(\frac{1}{n}\right) \log c_{e}
$$

Where $Q_{e}$ is the equilibrium dye concentration on adsorbent at any time $(\mathrm{mg} / \mathrm{g}), k_{f}$ is the Freundlich constant $(\mathrm{mg} / \mathrm{g}), \frac{1}{n}$ is the exponential constant, $\boldsymbol{C}_{e}$ is the equilibrium dye concentration in sample $(\mathrm{mg} / \mathrm{L})$.

Figures 10 - 13 show the plots of Langmuir and Freundlich isotherm models for the adsorption of Safranin$\mathrm{O}$ dye solution with respect to varying initial dye concentration(10-50) for both CSAC and PPAC respectively. The values of the adsorption parameters for Langmuir and Freundlich Isotherm Models are also shown in Table 1 for both CSAC and PPAC respectively. Based on the data presented in the Table (1), the correlation coefficients $\left(\mathrm{R}^{2}\right)$ obtained from the plots of Langmuir Isotherm Model fell within the range of 0.9981- 0.9992 for both CSAC and PPAC respectively. This implies that the model is suitable since it fitted well with the experimental data. This suggests that the adsorption of Safranin-O dye onto the CSAC and PPAC could be described as a monolayer adsorption and occurred on a homogenous surface. The maximum monolayer adsorption capacities were found to be $-60.194 \mathrm{mg} / \mathrm{g}$ for PPAC and $69.013 \mathrm{mg} / \mathrm{g}$ for CSAC respectively. Also, the values of $R_{L}$ were found to be 1.6667 for PPAC and 1.7065 for CSAC which implies that adsorption of Safranin-O dye onto both PPAC and 
CSAC was not favourable with respect to varying initial concentration. For Freundlich isotherm model, it could also be seen from the Table (1) that the correlation coefficients $\left(\mathrm{R}^{2}\right)$ fell within the range of $0.9995-0.9998$. This also signifies that the model is very good and well fitted with the observed data which implies that the adsorption process could be better be described by this model. This means that the adsorption occurred on a heterogeneous surface which may be attributed to the various active sites on the adsorbent surfaces [24]. The values of ( $n$ ) was found to be greater than one $(n>1)$ in all cases which implies that the adsorption process of Safranin-O onto the activated carbons were favourable.

The overall results of the adsorption isotherm analysis indicate that the adsorption of Safranin-O dye onto the two activated carbons exhibit both Langmuir and Freundlich isotherm models characteristics reflecting the presence of more than one kind of adsorbent-adsorbate surface interactions.

Table 1. Langmuir and Freundlich Paramaters.

\begin{tabular}{llllllll}
\hline Langmuir Model & \multicolumn{7}{c}{ Freundlich Model } \\
\hline Adsorbents & $\mathbf{B}(\mathbf{L} / \mathbf{m g})$ & $\mathbf{Q}_{\max }(\mathbf{m g} / \mathbf{g})$ & $\mathbf{R}_{\mathbf{L}}$ & $\mathbf{R}^{\mathbf{2}}$ & $\mathbf{K}_{\mathbf{f}}(\mathbf{m g} / \mathbf{g})$ & $\mathbf{n}$ & $\mathbf{R}^{\mathbf{2}}$ \\
\hline CSAC & -69.013 & -0.0207 & 1.7065 & 0.9992 & 1.1260 & 1.2532 & 0.9805 \\
PPAC & -60.914 & -0.0237 & 1.6667 & 0.9981 & 0.4331 & 1.9800 & 0.9805 \\
\hline
\end{tabular}

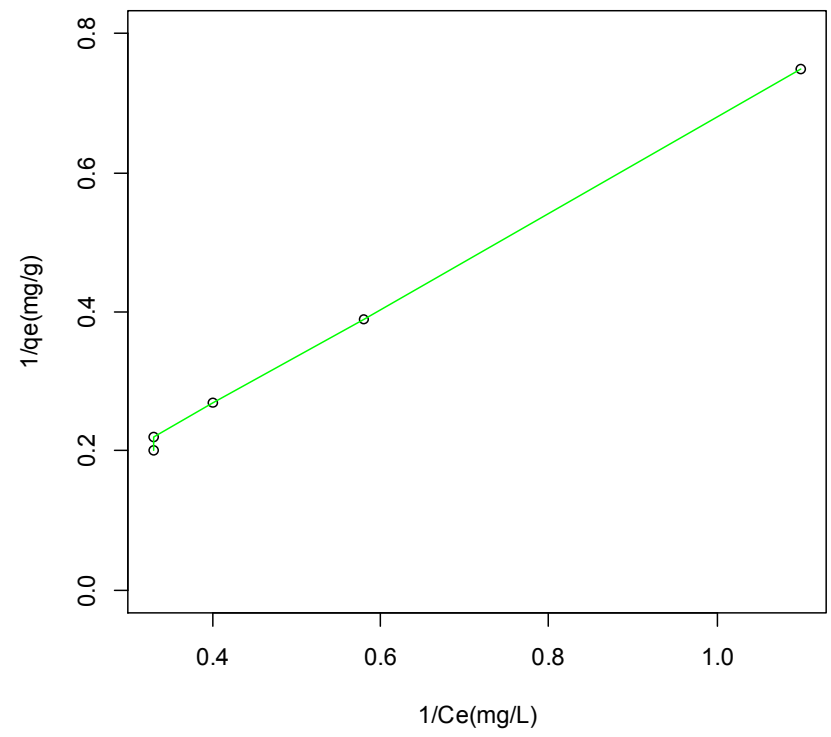

Figure 10. Langmuir Model for Adsorption of Safranin-O Dye onto PPAC.

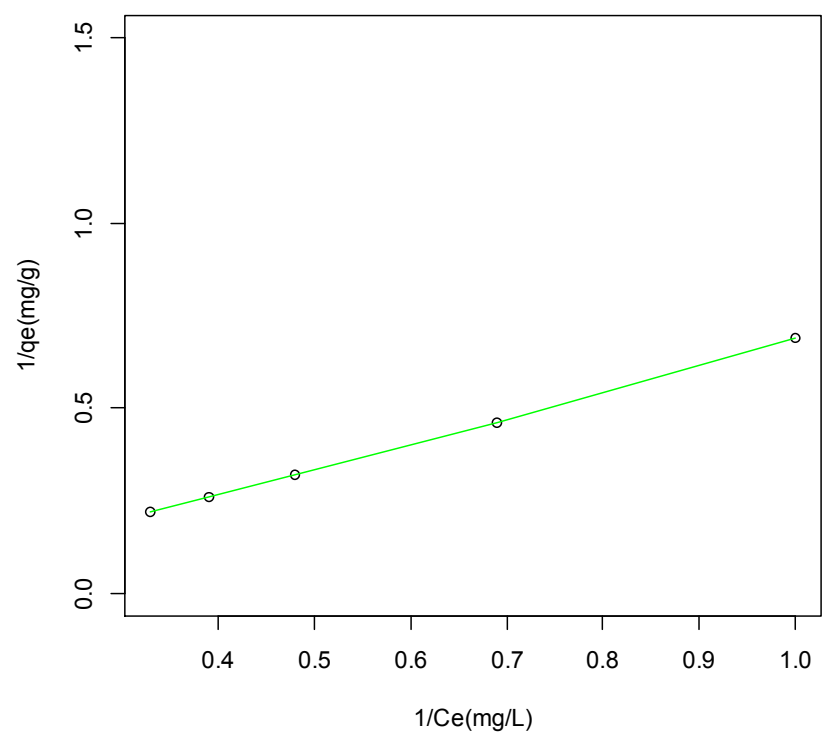

Figure 11. Langmuir Model for Adsorption of Safranin-O Dye onto CSAC.

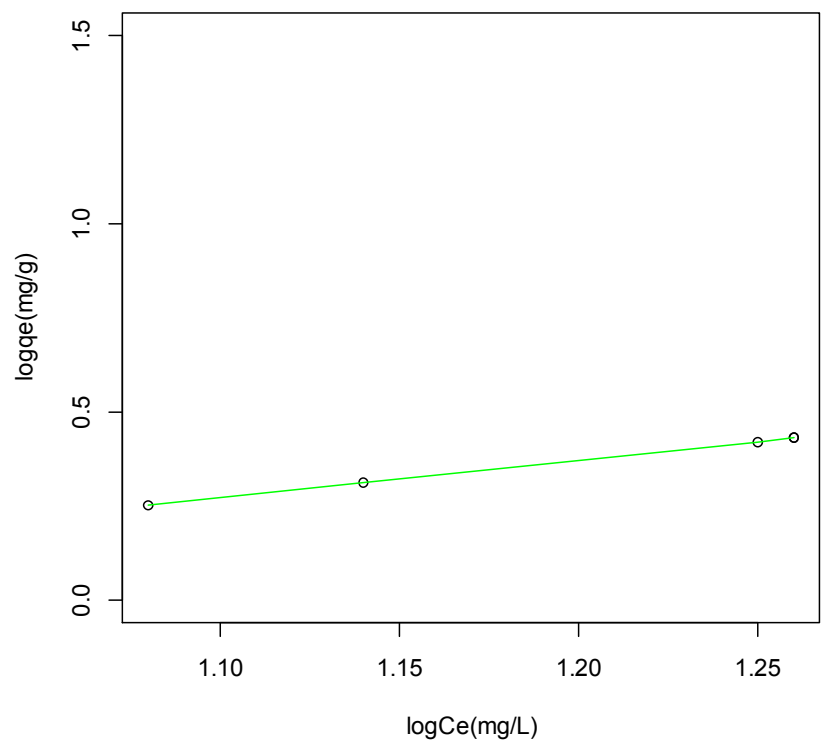

Figure 12. Freundlich Model for Adsorption of Safranin-O onto CSAC.

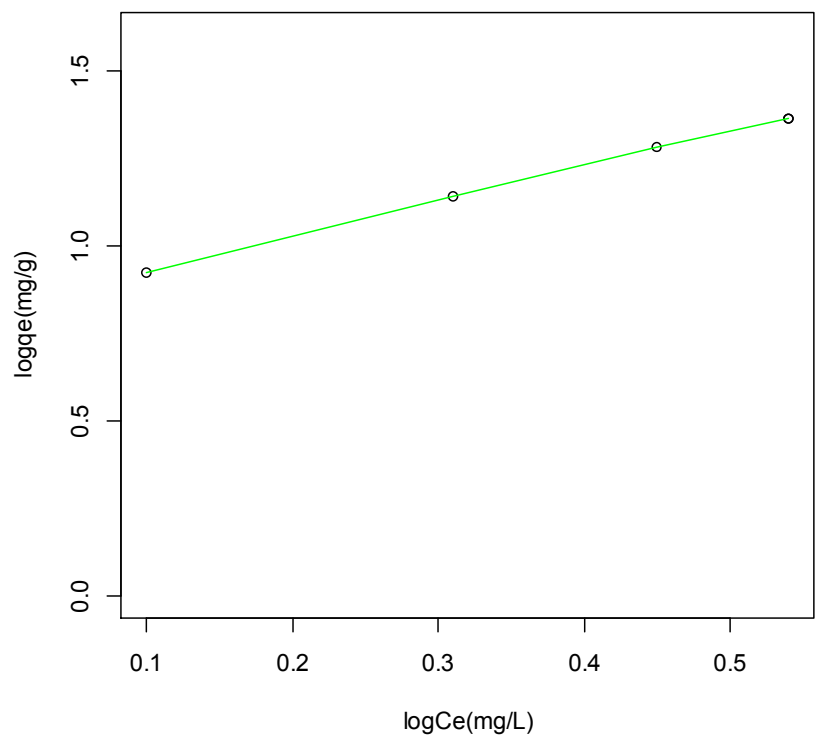

Figure 13. Freundlich Model for Adsorption of Safranin-O Dye onto PPAC. 


\subsection{Analysis of the Adsorption Kinetics}

Kinetic models are used to describe the dynamic nature of the adsorption process in terms of the order of rate constant [24]. In this study, pseudo-first-order and pseudo-secondorder kinetic models were applied. A linear form of pseudofirst-order model is given in equation (7) [25].

$$
\log \left(Q_{e}-Q_{t}\right)=\log Q_{e}-\frac{k_{1}}{2,303} t
$$

Where $Q_{e}$, is the equilibrium dye concentration on adsorbent at any time $(\mathrm{mg} / \mathrm{g}), Q_{t}$ is the amount of dye uptake $(\mathrm{mg} / \mathrm{g})$ at time, $\mathrm{t}(\mathrm{min})$ and $\boldsymbol{k}_{1}$ is the rate constant of pseudo-first-order sorption, $\left(\mathrm{min}^{-1}\right)$. A linear form of pseudosecond-order model is given in equation (8).

$$
\frac{1}{Q_{t}}=\frac{1}{k_{2} Q_{e}^{2}}+\frac{1}{Q_{e}} t
$$

Where $k_{2}$ is the equilibrium rate constant of pseudosecond-order (g/mg min), $Q_{t}$ is the amount of dye uptake $(\mathrm{mg} / \mathrm{g})$ at time, $\mathrm{t}(\mathrm{min}), Q_{e}$, is the equilibrium dye concentration on adsorbent at any time $(\mathrm{mg} / \mathrm{g})$ and $t$ is the contact time ( $\mathrm{min})$.

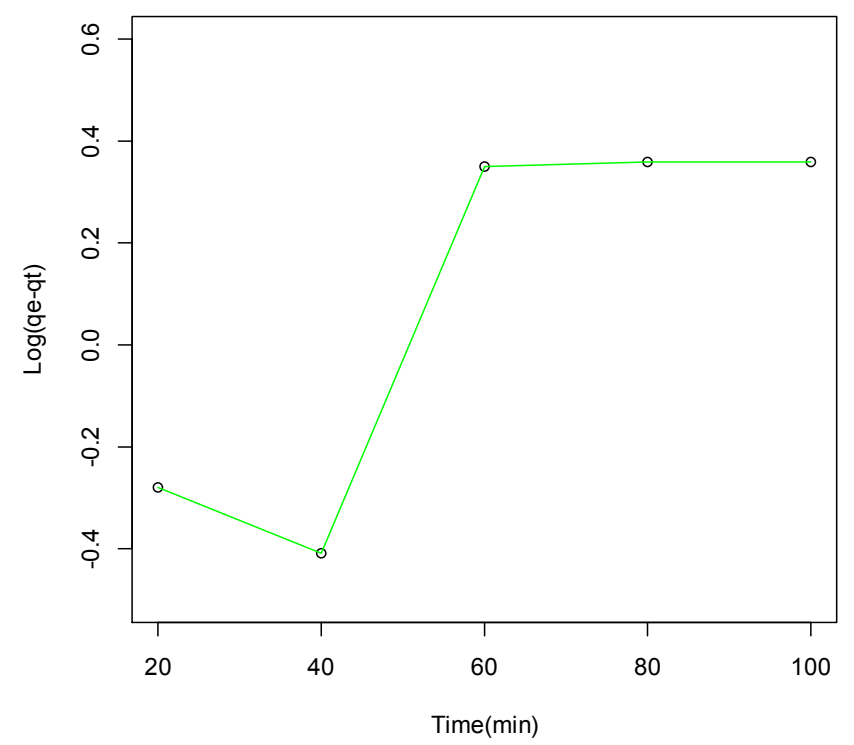

Figure 14. Plot of Pseudo First Order Kinetic Model for Adsorption of Safranin-O Dye Using CSAC.

Figures 14-17 show the plots of the two kinetic models while Table 2 shows the Kinetic model constants/parameters and correlation coefficients for the adsorption of Safranin-O dye solution using the two adsorbents. Based on the results obtained from the pseudo-first-order model plot, the values of correlation coefficients $\left(\mathrm{R}^{2}\right)$ were found to be within the range of $0.7524-0.7858$ for both adsorbents. Literature reveals that a suitable model is one that gives a perfect fit with the experimental data and is achieved when the value of $\mathrm{R}^{2}$ is within the range of 0.9 to 1.0 [26]. This implies that the model does not fit well with the observed data and might not be suitable to describe the dynamic nature of the adsorption process.

For pseudo-second-order model, the values of the correlation coefficients $\left(\mathrm{R}^{2}\right)$ were found to be within the range of $(0.9987-0.9999)$ indicating a very good model which means well fitted with the observed data. This suggests that the dynamic nature of the adsorption of Safanin-O dye onto the two activated carbons can best be described by pseudo-second-order model. Similar results were obtained by [26] on batch adsorption of basic dye using acid treated fiber char.

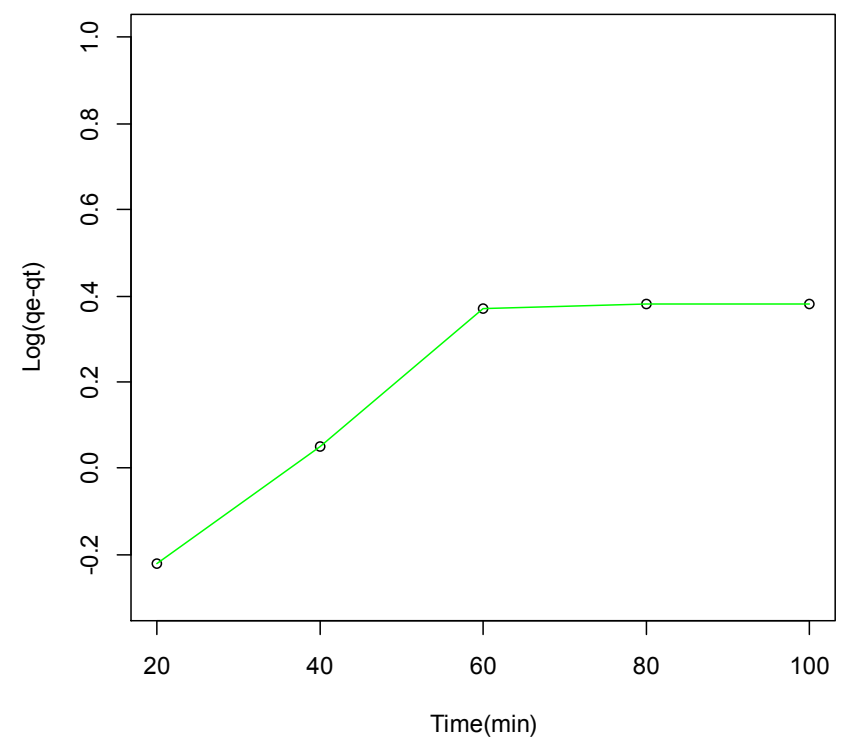

Figure 15. Plot of Pseudo First Order Kinetic Model for Adsorption of Safranin-O Dye Using PPAC.

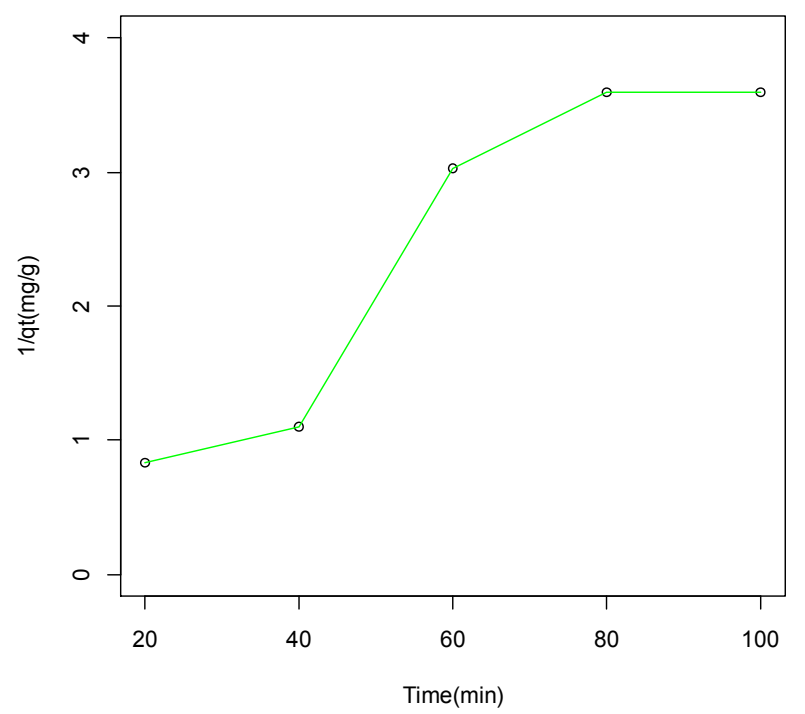

Figure 16. Plot of Pseudo Second Order Kinetic Model for the Adsorption of Safranin-O Dye Using CSAC. 


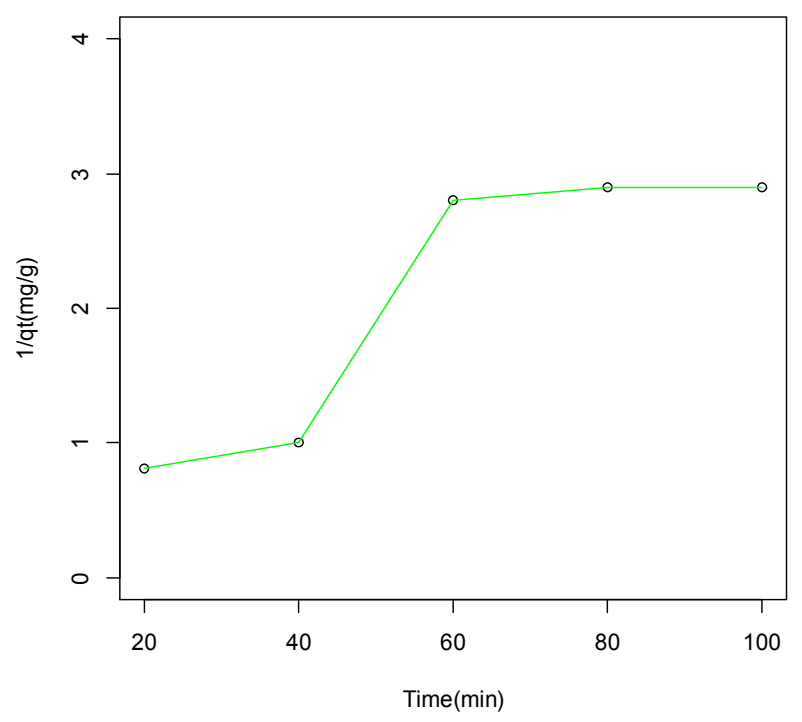

Figure 17. Plot of Pseudo Second Order Kinetic Model for the Adsorption of Safranin-ODye Using PPAC.

\subsection{Thermodynamic Analysis of the Adsorption Process}

The thermodynamic parameters were determined at various temperatures of $30,40,50$ and $60^{\circ} \mathrm{C}$ using Van't Hoff equations (9) and (10) respectively [26].

$$
\begin{gathered}
\ln \left(K_{d}\right)=\frac{\Delta S_{a d s}}{R}+\frac{\Delta H_{a d s}}{R T} \\
\Delta G_{a d s}=\Delta H_{a d s}-T \Delta S_{a d s}
\end{gathered}
$$

Where $K_{d}$, is the distribution coefficient at different temperatures (i.e. $\left.K_{d}=\mathrm{Ce}_{\mathrm{ads}} / \mathrm{Ce}_{\mathrm{s}}\right), \Delta G_{a d s}$ is the change in Gibbs free energies at different temperatures, $\mathrm{R}$ is the gas constant, $\Delta H$ ads is the change in enthalpy of the adsorption process and $\Delta S_{a d s}$ is the change in entropy of the adsorption process.

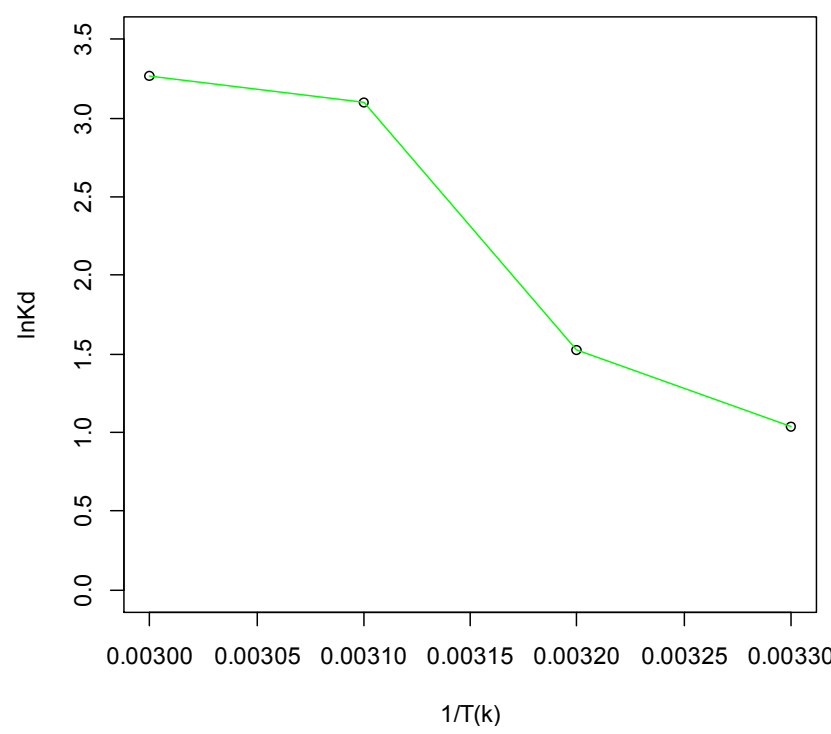

Figure 18. Van't Hoff Plot for the Safranin-O Dye Adsorption onto CSAC.

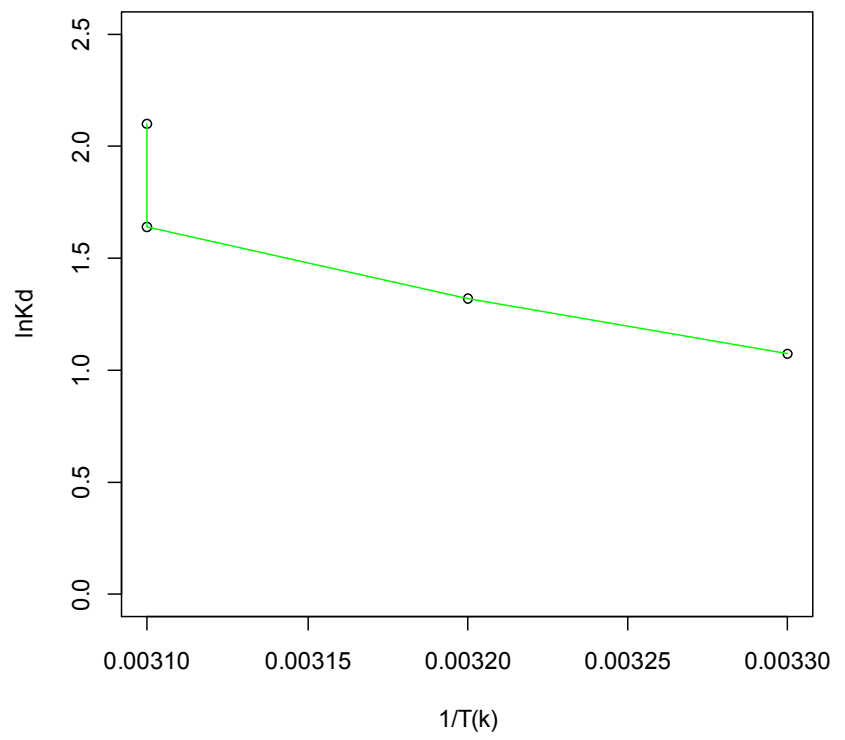

Figure 19. Van't Hoff Plot for the Safranin-O dye Adsorption onto PPAC.

\begin{tabular}{|c|c|c|c|c|c|c|}
\hline \multicolumn{7}{|c|}{ Kinetics Models } \\
\hline \multicolumn{3}{|c|}{ Pseudo- first order } & \multicolumn{4}{|c|}{ Pseudo-second order } \\
\hline Adsorbents & $Q_{\max }(\mathrm{mg} / \mathrm{g})$ & $\mathbf{R}^{2}$ & $K_{1}\left(\min ^{-1}\right)$ & $Q_{\max }(\mathrm{mg} / \mathrm{g})$ & $\mathbf{R}^{2}$ & $K_{2}(g / m g$ min) \\
\hline CSAC & 0.9980 & 0.7858 & -0.0260 & 24.870 & 0.9999 & 0.8000 \\
\hline PPAC & 1.0020 & 0.7833 & -0.0236 & 32.890 & 0.9984 & 0.0040 \\
\hline
\end{tabular}

Table 2. Kinetic Parameters and Correlation Coefficients for the Adsorption of Safranin-O.

The values of $\Delta S$ and $\Delta H$ were obtained from the intercept and slope using Van't Hoff plot of $\ln \mathrm{K}_{\mathrm{d}}$ against $\mathrm{I} / \mathrm{T}$ as shown in figures 18-19 respectively. Table 3 displays the values of the thermodynamic parameters of the adsorption process, the negative values of $\Delta G$ in both CSAC and PPAC imply that the adsorption process was thermodynamically feasible and spontaneous. Furthermore, the free energy change for the adsorption was greater than $-20 \mathrm{Kj} / \mathrm{mol}$ and less than zero in all cases.
This implies that the magnitude of $\Delta G$ values were in the range of multilayer adsorption which occurs by both physisorption and weak or partial chemisorptions [27]. This also, confirms the results of the adsorption isotherm which followed the Freundlich model. The value of $\Delta G$ became more negative with increasing temperature. This shows that increase in temperature was favourable for the removal of Safranin-O using the two adsorbents. Also from the Table $3, \Delta H$ values were observed to be 
negative in the two adsorbents which signify that the process was exothermic. The change in entropy $(\Delta S)$ values were observed to be positive at given temperatures implying that the degree of dispersion in the adsorption process increased with increase in temperature [28].

Table 3. Thermodynamic Parameters for the Adsorption of Safranin-O Dye Solution Using.

\begin{tabular}{lllll}
\hline \multicolumn{1}{l}{ CSAC, and PPAC } & & & & \\
\hline \multicolumn{1}{l}{ Thermodynamic parameters } & $\mathbf{T}(\mathbf{K})$ & $\Delta \mathbf{G}(\mathbf{K J} / \mathbf{m o l})$ & $\Delta \mathbf{S}(\mathbf{K J} / \mathbf{m o l} / \mathbf{K})$ & $\Delta \mathbf{H}(\mathbf{K J} / \mathbf{m o l})$ \\
\hline Adsorbents & 303 & -139.99 & 0.2351 & -68.750 \\
\hline CSAC & 313 & -142.30 & & \\
& 323 & -144.69 & & -34.380 \\
& 333 & -147.03 & 0.1219 & \\
PPAC & 303 & -71.330 & & \\
& 313 & -72.550 & & \\
& 323 & -73.770 & & \\
\hline
\end{tabular}

\section{Conclusion}

The effects of experimental factors such as initial dye concentration was found to be inversely proportional to percentage of Safranin-O dye removal while dosage, $\mathrm{pH}$ and contact time were directly proportional to the percentage of Safranin-O dye removal. Adsorption isotherms analysis indicate that the adsorption process exhibit both Langmuir and Freundlich isotherm model characteristic, reflecting the presence of more than one kind of adsorbent-adsorbate surface interaction. The adsorption kinetics models fitted well with pseudo-second-order kinetic model with high correlation coefficients ranging from(0.9984-0.9999). The thermodynamic study indicates that both $\Delta H$ and $\Delta G$ of the adsorption process in all cases were negative which implies the process is exothermic and is thermodynamically feasible and spontaneous. $\Delta S$ was positive signifying that the degree of dispersion in the adsorption process increased with increase in temperature. From the results obtained, it could be inferred that CSAC showed better adsorbent when compared with PPAC.

\section{Acknowledgements}

The authors sincerely appreciate the contributions of all the laboratory attendance in Chemistry Department, Nasarawa State University Keffi.

\section{References}

[1] Mohammed, M. A., Ibrahim, A. \& Shitu, A. (2014). Batch removal of hazardous Safranin-O in wastewater using pineapple peels as an agricultural waste based adsorbent. International Journal of Environmental Monitoring and Analysis, 2(3), 128-133.

[2] Malik, R. Ramteke, Wat, D. S. \& Erl, S. R. (2007). Adsorption of malachite green on groundnut shell waste based powdered activated carbon. Waste Management, 27(9), 1129-1138.
[3] Abdul Wahab, O., El-Nemr, A., El-Sikaily, A. \& Khaled, A. (2005). Use of rice husk for adsorption of direct dyes from aqueous solution: A case study of direct f. scarlet. Egypt. Journal of Aquatic Research, 3(1), 1110-1354.

[4] Abdullah, L. A. G., Mohd, Salleh, Siti, Mazlina, Mohd-Noor, J. M. \& Osman, M. R. (2005). Azo dye removal by adsorption using waste biomass: Sugarcane bagasse. Int. Journal of Engineering and Technology, 2(1), 8-13.

[5] Aci, F., Nebioglu, M., Arslan, M., Imamoglu, M., Zengin, M. \& Kucukislamoglu, M. (2008). Preparation of activated carbon from sugar beet molasses and adsorption of methylene blue. Environmental Bulletin, 17, 997-1001.

[6] Ansari, R. \& Mosayebzadeh, Z. (2010). Removal of basic dye methylene blue from aqueous solutions using sawdust and sawdust coated with polypyrrole. J. Iran. Chem. Soc., 7 (2), 339-350.

[7] Basar, C. A. (2006). Applicability of the various adsorption models of three dyes adsorption onto activated carbon prepared from waste apricot. J. Hazard Matter, 135, 232241.

[8] Bayazi, S. (2013). "Investigation of Safranin-Oadsorption on superparamagnetic iron oxide nanoparticles (SPION) and multi-wall carbon nanotube /SPION Composites," Desalin. Water Treat. pp. 1-10.

[9] Babel, S. \& Kurniawan, T. A. (2004). Cr(VI) Removal From Synthetic Wastewater using Coconut Shell Charcoal and Commercial Activated Carbon Modified with Oxidizing Agents and/or Chitosan. Chemosphere, 54, 951-967.

[10] Aksu, Z. (2001). Equilibrium and kinetics modeling of cadmium (II) biosorption by C. Vulgaris in a batch system: Effect of temperatures. Sep. and Purif. Tech., 21, 285-294.

[11] Arivoli, S. \& Thenkuzhali, M.( 2008). Kinetic, mechanistic, thermodynamic and equilibrium studies on the adsorption of rhodamine B by acid activated lowcost carbon. E-jour. Chem., 5(2), 187-200.

[12] Bansal, M., Singh, D., Garg, V. K. \& Rose, P. (2009). Equilibrium and kinetic studies on the adsorption of methylene blue using orange peels activated carbon. Int. Journal of Environmental Sciences and Engineering, 1(2), 108 -114 . 
[13] Kardivelu, K., Kavipriya, M., Karthika, C., Radhika, M. Vennilamani, N. a\& Pattabhi, S. (2003). Utilization of various agricultural wastes for activated carbon preparation and application for the removal of dyes and metal ions from aqueous solutions. Bioresoruce Technology, 87(1), 129-132.

[14] AOAC, (1990). Association of Official Analylical Chemist. Official Method of Analysis 15th Edn. Washinton D. C 147158

[15] Gupta, R. \& Mohapatra, H. (2003). Microial Biomass: An Economical Alternative for Removal of heavy metals from waste water. Indian Journal of Experimental Biology, 41, 945 -966 .

[16] Itodo, U. A. (2010). Comparative studies on the preparation, adsorption and evaluation of activated carbon from selected animals and agricultural wastes. a $\mathrm{PhD}$ thesis, Usmanu Danfodiyo University, Sokoto, Nigeria.

[17] Al-Othman, Z. A., Ali, R. \& Navshat, Mu.(2012). Hexavalent chromium removal from aqeuous medium by activated carbon prepared from peanut shell: Adsorption kinetics, equilibrium and thermodynamic studies. Chemical Engineering Journal, $184,238-247$.

[18] Andre, L. Cazetta, Alexandro, M. M., Vargas, Eurica, Nogami, M., Marcos, H. Kunita, Tais, L. Silva, et al.(2011). NaOH activated carbon of high surface area produced from coconut shell: Kinetic and equilibrium studies from the methylene blue adsorption. Chemical Engineering Journal, 174, 117-125.

[19] Emad, N. El-Qada, Stephen, J. Allen, Gavin, M. Walker (2006). Adsorption of methylene blue onto activated carbon produced from steam activated bituminous coal: A study of equilibrium adsorption isotherm. Chemical Engineering Journal, 124, $103-110$.

[20] Ahmad, A. A. \& Hameed, B. H. (2009). Reduction of COD and colour of dyeing effluent from a cotton textile mill by adsorption onto bamboo-based activated carbon. J. Hazard. Mater. 172, - 154 .
[21] Nadeem, R., Hanif, M. A., Shaheen, F., Perveen, S., Zafar, M. N., \& Iqbal, T. (2008). Physical and chemical modification of distillery sludge for $\mathrm{Pb}(\mathrm{II})$ biosorption. Journal of Hazardous Material, 150, $335-342$.

[22] Okoli, C. A., Onukwuli, O. D., Okey-Onyesolu, C. F. \& Okoye, C. C. (2015). Adsorptive removal of dyes from synthetic wastewater using activated carbon from tamarind seed. European Scientific Journal, 11(18), 1857-1859.

[23] Robinson, T., Chandran, B. \& Nigam, P. (2002). Studies on desorption of individual textile dyes and synthetic dye effluent from dye adsorbed-agricultural residues using solvents. Bioresour Technol, 84, 299-301.

[24] Savas, Sener (2008). Use of solid waste of the soda ash plant as an adsorbent for the removal of anionic dye: Equilibrium and kineticstudies. Chemical Engineering Journal, 138, 207 214.

[25] Ponnusami, V., Vikiram, S. \& Srivastava, S. N. (2008). Guava psidium guajava leaf powder: Novel adsorbent for removal of methylene blue from aqueous solutions. J Hazard Maters, 152, 276-286.

[26] Edokpayi, O., Osemwenkhae, O; Ayodele, B. V; Ossai, J., Fadilat, S. A., Ogbeide, S. E(2018). Batch Adsorption Study of Methylene Blue in Aqueous Solution using Activated Carbons from Rice Husk and Coconut Shell. J. Appl. Sci. Environ. Manage, Vol. 22(5)63-635.

[27] Dalia, K. Maamoud, Mohammad, Amran, Mohasalleh, W. Azlina, Wari, A. Karima, A. Idris \& Zurina, Z. Abidin (2012). Batch adsorption of basic dye using acid treated fibre char: Equilibrium, kinetic and thermodynamic studies. Chemical Engineering Journal 181, 449 - 457.

[28] Ejikeme, M. Ebere, Ejikeme, P. C. N., Abalu, Benjamin. N. (2014). Equilibrium, kinetics and thermodynamic studies on MB adsorption using hamburger seed shell activated carbon. International Journal of Engineering \& Technology, 14(3), 74-83. 